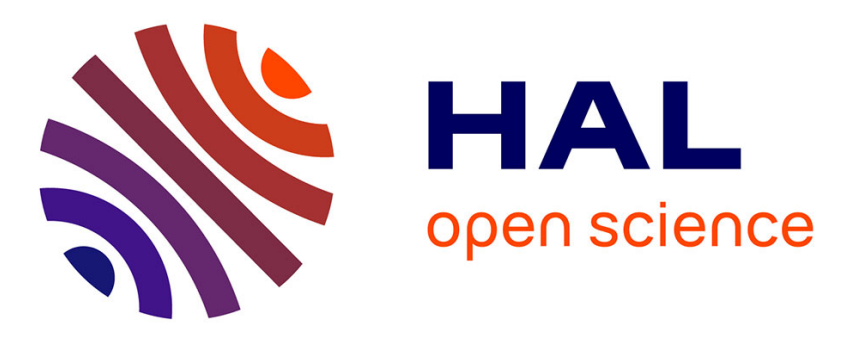

\title{
Determination of a Dynamic Feasible Workspace for Cable-Driven Parallel Robots
}

Lorenzo Gagliardini, Marc Gouttefarde, Stéphane Caro

\section{To cite this version:}

Lorenzo Gagliardini, Marc Gouttefarde, Stéphane Caro. Determination of a Dynamic Feasible Workspace for Cable-Driven Parallel Robots. J Lenarčič; J.P Merlet. Advances in Robot Kinematics 2016, 4, pp.361-370, 2018, Springer Proceedings in Advanced Robotics, 978-3-319-56802-7. 10.1007/978-3-319-56802-7_38. hal-01758038

\section{HAL Id: hal-01758038 \\ https://hal.science/hal-01758038}

Submitted on 4 Apr 2018

HAL is a multi-disciplinary open access archive for the deposit and dissemination of scientific research documents, whether they are published or not. The documents may come from teaching and research institutions in France or abroad, or from public or private research centers.
L'archive ouverte pluridisciplinaire HAL, est destinée au dépôt et à la diffusion de documents scientifiques de niveau recherche, publiés ou non, émanant des établissements d'enseignement et de recherche français ou étrangers, des laboratoires publics ou privés. 


\title{
Determination of a Dynamic Feasible Workspace for Cable-Driven Parallel Robots
}

\author{
L. Gagliardini, M. Gouttefarde, and S. Caro
}

\begin{abstract}
The dynamic equilibrium of the moving platform of a cable-driven parallel robot can be investigated by means of the Dynamic Feasible Workspace (DFW), which is the set of dynamic feasible moving platform poses. A pose is said to be dynamic feasible if a prescribed set of moving platform accelerations is feasible, with cable tensions lying in between given lower and upper bounds. This paper introduces an extended version of the DFW with respect to the one usually considered in the literature. Indeed, the improved DFW introduced in this paper takes into account: (i) The inertia of the moving platform; (ii) The external wrenches applied on the moving platform and (iii) The centrifugal and the Coriolis forces corresponding to a constant moving platform twist. Finally, the static, wrench-feasible, dynamic and improved dynamic workspaces of a spatial cable-suspended parallel robot are plotted in order to compare their sizes.
\end{abstract}

Key words: Cable-Driven Parallel Robots, Workspace Analysis, Dynamic Feasible Workspace

\section{Introduction}

Several industries, e.g. the naval and renewable energy industries, are facing the necessity to manufacture novel products of large dimensions and complex shapes.

L. Gagliardini

Robotics Team, IRT Jules Verne, Chemin du Chaffault, 44340, Bouguenais, France e-mail: lorenzo.gagliardini@irt-jules-verne.fr

M. Gouttefarde

CNRS-LIRMM, 161 rue Ada, 34392, Montpellier Cedex 05, France e-mail:

marc.gouttefarde@lirmm.fr

S. Caro

CNRS-IRCCyN, 1, rue de la Noë, 44321, Nantes Cedex 03, France e-mail: stephane.caro@irccyn.ec-nantes.fr 


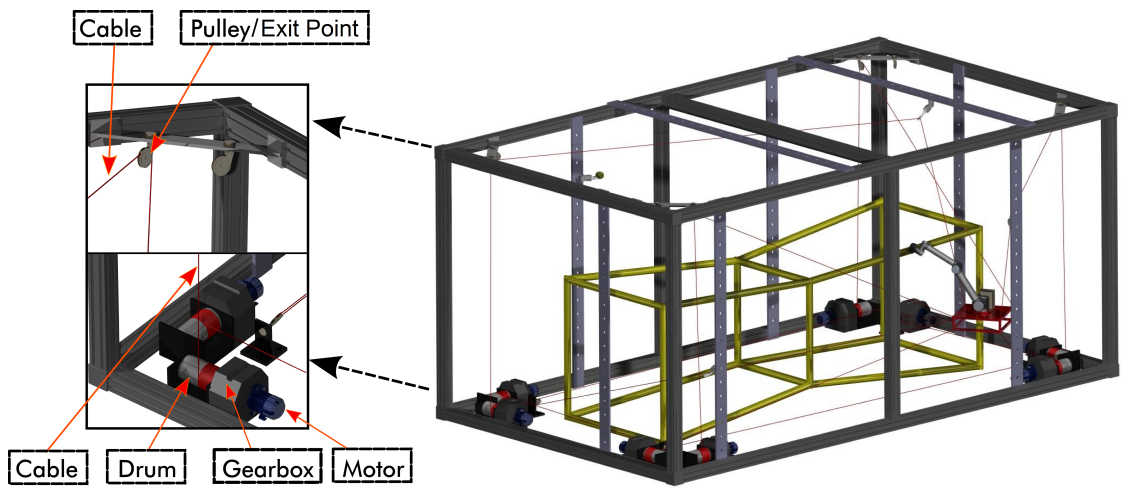

Fig. 1 Example of a CDPR design created in the framework of the IRT JV CAROCA project.

In order to ease the manufacturing of such products, the IRT Jules Verne promoted the investigation of new technologies. In this context, the CAROCA project aims at investigating the performance of Cable Driven Parallel Robots (CDPRs) to manufacture large products in cluttered industrial environments [5,6]. CDPRs are a particular class of parallel robots whose moving platform is connected to the robot fixed base frame by a number of cables as illustrated in Fig. 1. CDPRs have several advantages such as a high payload-to-weight ratio, a potentially very large workspace, and possibly reconfiguration capabilities.

The equilibrium of the moving platform of a CDPR is classically investigated by analyzing the CDPR workspace. In serial and rigid-link parallel robots, the workspace is commonly defined as the set of end-effector poses where a number of kinematic constraints are satisfied. In CDPRs, the workspace is usually defined as the set of poses where the CDPR satisfies one or more conditions including the static or the dynamic equilibrium of the moving platform, with the additional constraint of non-negative cable tensions. Several workspaces and equilibrium conditions have been studied in the literature.

The first investigations focused on the static equilibrium and the Wrench Closure Workspace (WCW) of the moving platform, e.g. [4, 9, 14-16]. Since cables can only pull on the moving platform, a pose belongs to the WCW if and only if any wrench can be applied by means of non-negative cable tensions. Feasible equilibria of the moving platform can also be analyzed using the Wrench Feasible Workspace (WFW) [2, 3, 8]. By definition, the WFW is the set of wrench feasible platform poses where a pose is wrench feasible when the cables can balance a given set of external moving platform wrenches while maintaining the cable tensions in between given lower and upper bounds. The Static Feasible Workspace (SFW) is a special case of the WFW, where the sole wrench induced by the moving platform weight has to be balanced [13]. The lower cable tension bound, $\tau_{\min }$, is defined in order to prevent the cables from becoming slack. The upper cable tension bound, $\tau_{\max }$, is defined in order to prevent the CDPR from being damaged. 
The dynamic equilibrium of the moving platform can be investigated by means of the Dynamic Feasible Workspace (DFW). By definition, the DFW is the set of dynamic feasible moving platform poses. A pose is said to be dynamic feasible if a prescribed set of moving platform accelerations is feasible, with cable tensions lying in between given lower and upper bounds. The concept of dynamic workspace has already been investigated in [1] for planar CDPRs. Barrette et al. solved the dynamic equations of a planar CDPR analytically, providing the possibility to compute the boundary of the DFW. This strategy cannot be directly applied to spatial CDPRs due to the complexity of their dynamic model. In 2014, Kozlov studied in [12] the possibility to investigate the DFW by using a tool developed by Guay et al. for the analysis of the WFW [11]. However, the dynamic model proposed by Kozlov considers the moving platform as a point mass, neglecting centrifugal and Coriolis forces.

This paper deals with a more general definition of the DFW. With respect to the definitions proposed in $[1,12]$, the DFW considered in the present paper takes into account: (i) The inertia of the moving platform; (ii) The external wrenches applied on the moving platform; (iii) The centrifugal and the Coriolis forces corresponding to a given moving platform twist. The Required Wrench Set (RWS), defined here as the set of wrenches that the cables have to apply on the moving platform in order to satisfy its dynamic equilibrium, is calculated as the sum of these three contributions to the dynamic equilibrium. Then, the corresponding DFW is computed by means of the algorithm presented in [10] to analyze the WFW.

\section{Dynamic Model}

The CDPR dynamic model considered in this paper consists of the dynamics of the moving platform. A dynamic model taking into account the dynamics of the winches could also be considered but is not used here due to space limitations. Additionally, assuming that the diameters of the cables and the pulleys are small, the dynamics of the pulleys and the cables is neglected.

The dynamic equilibrium of the moving platform is described by the following equation

$$
\mathbf{W} \tau-\mathbb{I}_{p} \ddot{\mathbf{p}}-\mathbf{C} \dot{\mathbf{p}}+\mathbf{w}_{e}+\mathbf{w}_{g}=0
$$

where $\mathbf{W}$ is the wrench matrix that maps the cable tension vector $\tau$ into a platform wrench, and

$$
\dot{\mathbf{p}}=\left[\begin{array}{c}
\dot{\mathbf{t}} \\
\omega
\end{array}\right] \quad \ddot{\mathbf{p}}=\left[\begin{array}{c}
\ddot{\mathbf{t}} \\
\alpha
\end{array}\right],
$$

where $\dot{\mathbf{t}}=\left[\dot{x}_{x}, \dot{y}_{y}, \dot{t}_{z}\right]^{\mathrm{T}}$ and $\ddot{\mathbf{t}}=\left[\ddot{t}_{x}, \ddot{t}_{y}, \ddot{t}_{z}\right]^{\mathrm{T}}$ are the vectors of the moving platform linear velocity and acceleration, respectively, while $\omega=\left[\omega_{x}, \omega_{y}, \omega_{z}\right]^{\mathrm{T}}$ and $\alpha=\left[\alpha_{x}, \alpha_{y}, \alpha_{z}\right]^{\mathrm{T}}$ are the vectors of the moving platform angular velocity and acceleration, respectively. 
The external wrench $\mathbf{w}_{e}$ is a 6-dimensional vector expressed in the fixed reference frame $\mathscr{F}_{b}$ and takes the form

$$
\mathbf{w}_{e}=\left[\mathbf{f}_{e}^{\mathrm{T}}, \mathbf{m}_{e}^{\mathrm{T}}\right]^{\mathrm{T}}=\left[f_{x}, f_{y}, f_{z}, m_{x}, m_{y}, m_{z}\right]^{\mathrm{T}}
$$

$f_{x}, f_{y}$ and $f_{z}$ are the $x, y$ and $z$ components of the external force vector $\mathbf{f}_{e} . m_{x}, m_{y}$ and $m_{z}$ are the $x, y$ and $z$ components of the external moment vector $\mathbf{m}_{e}$, respectively. The components of the external wrench $\mathbf{w}_{e}$ are assumed to be bounded as follows

$$
\begin{gathered}
f_{\min } \leq f_{x}, f_{y}, f_{z} \leq f_{\max } \\
m_{\min } \leq m_{x}, m_{y}, m_{z} \leq m_{\max }
\end{gathered}
$$

According to (4) and (5), the set $\left[\mathbf{w}_{e}\right]_{r}$, called the Required External Wrench Set (REWS), that the cables have to balance is a hyper-rectangle.

The Center of Mass (CoM) of the moving platform, $G$, may not coincide with the origin of the frame $\mathscr{F}_{p}$ attached to the platform. The mass of the platform being denoted by $M$, the wrench $\mathbf{w}_{g}$ due to the gravity acceleration $\mathbf{g}$ is defined as follows

$$
\mathbf{w}_{g}=\left[\begin{array}{c}
M \mathbf{I}_{3} \\
\mathbf{M} \hat{\mathbf{S}}_{p}
\end{array}\right] \mathbf{g}
$$

where $\mathbf{I}_{3}$ is the $3 \times 3$ identity matrix, $\mathbf{M S}_{p}=\mathbf{R}\left[M x_{p}, M y_{p}, M z_{p}\right]^{\mathrm{T}}$ is the first momentum of the moving platform defined with respect to frame $\mathscr{F}_{b}$. The vector $\mathbf{S}_{p}=\left[x_{p}, y_{p}, z_{p}\right]^{\mathrm{T}}$ defines the position of $G$ in frame $\mathscr{F}_{p}$. M. $\hat{\mathbf{S}}_{p}$ is the skew-symmetric matrix associated to $\mathbf{M S}_{p}$.

The matrix $\mathbb{I}_{p}$ represents the spatial inertia of the platform

$$
\mathbb{I}_{p}=\left[\begin{array}{cc}
M \mathbf{I}_{3} & -\mathbf{M} \hat{\mathbf{S}}_{p} \\
\mathbf{M} \hat{\mathbf{S}}_{p} & \mathbf{I}_{p}
\end{array}\right]
$$

where $\mathbf{I}_{p}$ is the inertia tensor matrix of the moving platform, which can be computed by the Huygens-Steiner theorem from the moving platform inertia tensor, $\mathbf{I}_{g}$, defined with respect to the platform $\mathrm{CoM}$

$$
\mathbf{I}_{p}=\mathbf{R} \mathbf{I}_{g} \mathbf{R}^{\mathrm{T}}-\frac{\mathbf{M} \hat{\mathbf{S}}_{p} \mathbf{M} \hat{\mathbf{S}}_{p}}{M}
$$

$\mathbf{R}$ is the rotation matrix defining the moving platform orientation and $\mathbf{C}$ is the matrix of the centrifugal and Coriolis wrenches, defined as

$$
\mathbf{C} \dot{\mathbf{p}}=\left[\begin{array}{c}
\hat{\omega} \hat{\omega} \mathbf{M} \mathbf{S}_{p} \\
\hat{\omega} \mathbf{I}_{p} \omega
\end{array}\right]
$$

where $\hat{\omega}$ is the skew-symmetric matrix associated to $\omega$. 


\section{Dynamic Feasible Workspace}

\subsection{Standard Dynamic Feasible Workspace}

Studies on the DFW have been realised by Barrette et al. in [1]. The boundaries of the DFW have been computed for a generic planar CDPR developing the equations of its dynamic model. Since this method cannot be easily extended to spatial CDPRs, Kozlov proposed to use the method described in [11] in order to compute the DFW of a fully constrained CDPR [12]. The proposed method takes into account the cable tension limits $\tau_{\min }$ and $\tau_{\max }$ in checking the feasibility of the dynamic equilibrium of the moving platform for the following bounded sets of accelerations

$$
\begin{gathered}
\ddot{\mathbf{t}}_{\text {min }} \leq \ddot{\mathbf{t}} \leq \ddot{\mathbf{t}}_{\text {max }} \\
\alpha_{\text {min }} \leq \alpha \leq \alpha_{\text {max }}
\end{gathered}
$$

where $\ddot{\mathbf{t}}_{\text {min }}, \ddot{\mathbf{t}}_{\text {max }}, \alpha_{\text {min }}, \alpha_{\text {max }}$ are the bounds on the moving platform linear and rotational accelerations. These required platform accelerations define the so-called Required Acceleration Set (RAS), $[\ddot{\mathbf{p}}]_{r}$. The RAS can be projected into the wrench space by means of matrix $\mathbb{I}_{p}$, defined in (7). The set of wrenches $\left[\mathbf{w}_{d}\right]_{r}$ generated by this linear mapping is defined as the Required Dynamic Wrench Set (RDWS). No external wrench is applied to the moving platform. Accordingly, the DFW is defined as follows

Definition 1. A moving platform pose is said to be dynamic feasible when the moving platform of the CDPR can reach any acceleration included in $[\ddot{\mathbf{p}}]_{r}$ according to cable tension limits expressed by $[\tau]_{a}$. The Dynamic Feasible Workspace is then the set of dynamic feasible poses, $[\mathbf{p}]_{D F W}$.

$$
[\mathbf{p}]_{D F W}=\left\{(\mathbf{t}, \mathbf{R}) \in \mathrm{R}^{3} \times S O(3): \forall \ddot{\mathbf{p}} \in[\ddot{\mathbf{p}}]_{r}, \exists \tau \in[\tau]_{a} \text { s.t. } \mathbf{W} \tau-\mathbf{A} \ddot{\mathbf{p}}=0\right\}
$$

In the definition above, the set of Admissible Cable Tensions (ACT) is defined as

$$
[\tau]_{a}=\left\{\tau \mid \tau_{\min } \leq \tau_{i} \leq \tau_{\max }, i=1, \ldots, m\right\}
$$

\subsection{Improved Dynamic Feasible Workspace}

The DFW described in the previous section has several limitations. The main drawback is associated to the fact that the proposed DFW takes into account neither the external wrenches applied to the moving platform nor its weight. Furthermore, the model used to verify the dynamic equilibrium of the moving platform neglects the Coriolis and the centrifugal wrenches associated to the CDPR dynamic model. 
At a given moving platform pose, the cable tensions should compensate both the contribution associated to the REWS, $\left[\mathbf{w}_{e}\right]_{r}$, and the RDWS, $\left[\mathbf{w}_{d}\right]_{r}$. The components of the REWS are bounded according to (4) and (5) while the components of the RDWS are bounded according to (10) and (11).

The dynamic equilibrium of the moving platform is described by (1), where $\mathbf{C}$ is related to the Coriolis and centrifugal forces of the moving platform and $\mathbf{w}_{g}$ to its weight. These terms depend only on the pose and the twist of the moving platform. For given moving-platform pose and twist, these terms are constant.

Therefore, the DFW definition can be modified as follows.

Definition 2. A moving platform pose is said to be dynamic feasible when, for a given twist $\dot{\mathbf{p}}$, the CDPR can balance any external wrench $\mathbf{w}_{e}$ included in $\left[\mathbf{w}_{e}\right]_{r}$, while the moving platform can assume any acceleration $\ddot{\mathbf{p}}$ included in $[\ddot{\mathbf{p}}]_{r}$. The Dynamic Feasible Workspace is the set of dynamic feasible poses, $[\mathbf{p}]_{D F W}$.

$$
[\mathbf{p}]_{D F W}: \forall \mathbf{w}_{e} \in\left[\mathbf{w}_{e}\right]_{r}, \forall \ddot{\mathbf{p}} \in[\ddot{\mathbf{p}}]_{r}, \exists \tau \in[\tau]_{a} \text { s.t. } \mathbf{W} \tau-\mathbb{I}_{p} \ddot{\mathbf{p}}-\mathbf{C} \dot{\mathbf{p}}+\mathbf{w}_{e}+\mathbf{w}_{g}=0
$$

In this definition, we may note that the feasibility conditions are expressed according to three wrench space sets. The first set, $\left[\mathbf{w}_{d}\right]_{r}$, can be computed by projecting the vertices of $[\ddot{\mathbf{p}}]_{r}$ into the wrench space. For a 3 -dimensional case study (6 DoF case), $[\ddot{\mathbf{p}}]_{r}$ consists of 64 vertices. The second component, $\left[\mathbf{w}_{e}\right]_{r}$, consists of 64 vertices as well. Considering a constant moving platform twist, the last component of the dynamic equilibrium, $\mathbf{w}_{c}=\left\{\mathbf{C} \dot{\boldsymbol{p}}+\mathbf{w}_{g}\right\}$, is a constant wrench. The composition of these sets generates a polytope, $[\mathbf{w}]_{r}$, defined as the Required Wrench Set (RWS). $[\mathbf{w}]_{r}$ can be computed as the convex hull of the Minkowski sum over $\left[\mathbf{w}_{e}\right]_{r},\left[\mathbf{w}_{d}\right]_{r}$ and $\mathbf{w}_{c}$, as illustrated in Fig. 2:

$$
[\mathbf{w}]_{r}=\left[\mathbf{w}_{e}\right]_{r} \oplus\left[\mathbf{w}_{d}\right]_{r} \oplus \mathbf{w}_{c}
$$

Thus, Def. 2 can be rewritten as a function of $[\mathbf{w}]_{r}$.

Definition 3. A moving platform pose is said to be dynamic feasible when the CDPR can balance any wrench $\mathbf{w}$ included in $[\mathbf{w}]_{r}$. The Dynamic Feasible Workspace is the set of dynamic feasible poses, $[\mathbf{p}]_{D F W}$.

$$
[\mathbf{p}]_{D F W}: \forall \mathbf{w} \in[\mathbf{w}]_{r}, \exists \tau \in[\tau]_{a} \quad \text { s.t. } \quad \mathbf{W} \tau-\mathbb{I}_{p} \ddot{\mathbf{p}}+\mathbf{w}_{e}+\mathbf{w}_{c}=0
$$

The mathematical representation in (16) is similar to the one describing the WFW. As a matter of fact, from a geometrical point of view, a moving platform pose will be dynamic feasible if $[\mathbf{w}]_{r}$ is fully included in $[\mathbf{w}]_{a}$

$$
[\mathbf{w}]_{r} \subseteq[\mathbf{w}]_{a}
$$

Consequently, the dynamic feasibility of a pose can be verified by means of the hyperplane shifting method $[3,10,11]$. The distances between the facets of the avail- 


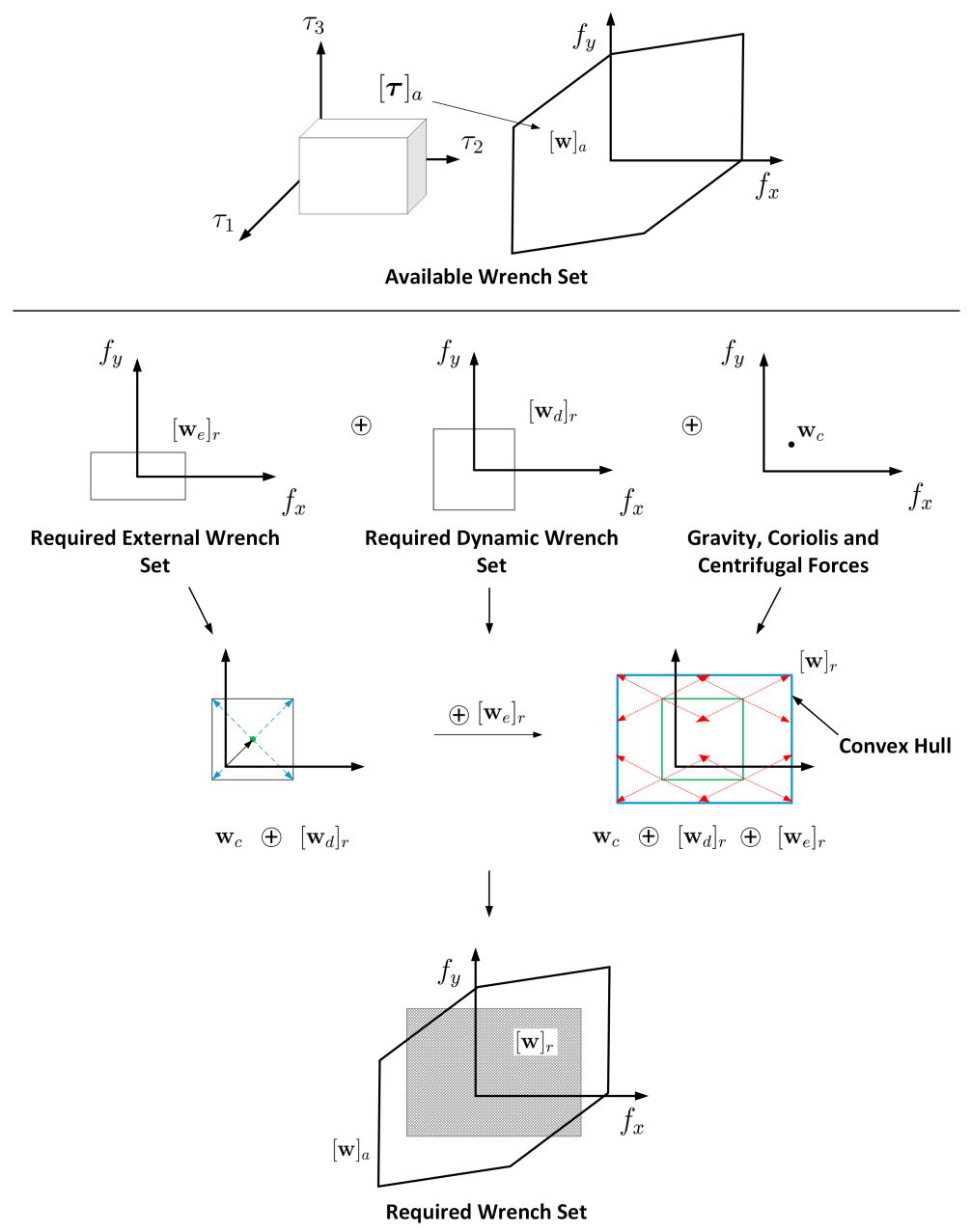

Fig. 2 Computation of the RWS $[\mathbf{w}]_{r}$. Example of a planar CDPR with 3 actuators and 2 translational DoF.

able wrench set, $[\mathbf{w}]_{a}$, and the vertices of the RWS, $[\mathbf{w}]_{r}$, is verified according to the following inequality

$$
\mathbf{C w}_{r} \leq \mathbf{d}_{d}, \quad \forall \mathbf{w} \in[\mathbf{w}]_{r}
$$

\section{Case Study}

This section aims at comparing the SFW, WFW, DFW and Improved DFW (IDFW) of the spatial suspended CDPR illustrated in Fig. 3. It has the layout the CoGiRo 




Fig. 3 Layout of the CoGiRo cable-suspended parallel robot [7] with the size of the IRT JV CAROCA prototype.

robot [7] and the size of the IRT JV CAROCA prototype. The robot consists of a moving platform connected to the fixed base frame by $m=8$ cables. It is $7 \mathrm{~m}$ long, $4 \mathrm{~m}$ width and $3.5 \mathrm{~m}$ high. The maximum cable tension is equal to $6990 \mathrm{~N}$. The moving platform consists of a parallelepiped. Its width, $w_{p}$, its length, $l_{p}$, and its height, $h_{p}$, are equal to $20 \mathrm{~cm}, 20 \mathrm{~cm}$ and $25 \mathrm{~cm}$, respectively. The mass of the moving platform, $M$, is equal to $100 \mathrm{~kg}$. In the proposed case study, the CoM of the platform, $G$, does not coincide with the origin $O_{p}$ of frame $\mathscr{F}_{p}$, being $\mathbf{S}=[1 \mathrm{~cm}, 1 \mathrm{~cm}, 1 \mathrm{~cm}]^{\mathrm{T}}$.

The volume inside the base frame has been discretized homogeneously into $n_{p}=$ 882 points. Each point has been analysed in order to verify if the corresponding poses of the CDPR belong to the improved DFW. The analysis has been performed assuming that the moving platform is aligned with respect to the axes of frame $\mathscr{F}_{b}$. The linear velocity of the moving platform is equal to $\dot{\mathbf{t}}=[1 \mathrm{~m} / \mathrm{s}, 1 \mathrm{~m} / \mathrm{s}, 1 \mathrm{~m} / \mathrm{s}]^{\mathrm{T}}$ and its angular velocity is equal to $\omega=[0.05 \mathrm{rad} / \mathrm{s}, 0.05 \mathrm{rad} / \mathrm{s}, 0.05 \mathrm{rad} / \mathrm{s}]^{\mathrm{T}}$. The external wrenches acting on the moving platform are bounded as follows:

$$
\begin{aligned}
& -100 \mathrm{~N} \leq f_{x}, f_{y}, f_{z} \leq 100 \mathrm{~N} \\
& -1 \mathrm{Nm} \leq m_{x}, m_{y}, m_{z} \leq 1 \mathrm{Nm}
\end{aligned}
$$

Similarly, the range of accelerations of the moving platform is limited according to the following inequalities:

$$
\begin{aligned}
-2 \mathrm{~m} / \mathrm{s}^{2} & \leq \ddot{t}_{x}, \ddot{t}_{y}, \ddot{t}_{z} \leq 2 \mathrm{~m} / \mathrm{s}^{2} \\
-0.1 \mathrm{rad} / \mathrm{s}^{2} & \leq \alpha_{x}, \alpha_{y}, \alpha_{z} \leq 0.1 \mathrm{rad} / \mathrm{s}^{2}
\end{aligned}
$$

For the foregoing conditions, the improved DFW of the CDPR covers the $47.96 \%$ of its volume. Figure 4(a) illustrates the improved DFW of the CDPR under study.

The results have been compared with respect to the dynamic feasibility conditions described by Def. 1. By considering only the weight and the inertia of the moving platform, the DFW covers the $63.27 \%$ of the volume occupied by the DFW, 


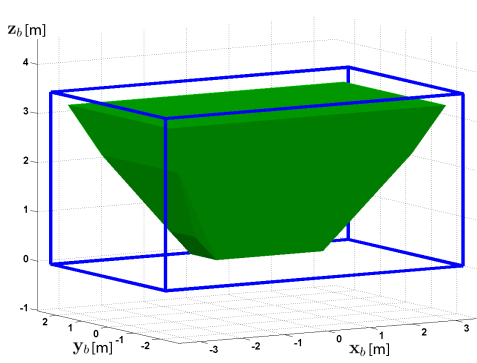

(a)

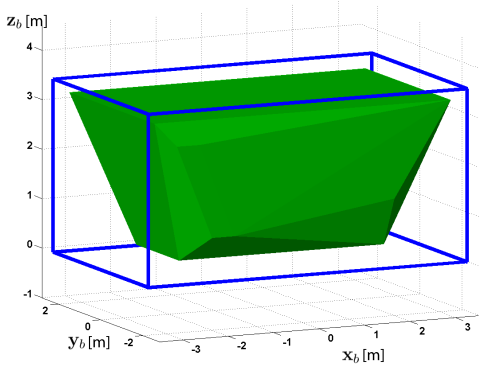

(b)

Fig. 4 (a) Improved DFW and (b) DFW of the CDPR under study covering $47.96 \%$ and $63.27 \%$ of its volume, respectively.

Table 1 Comparison of $S F W, W F W, D F W$ and $I D F W$ of the CDPR under study.

\begin{tabular}{c|cccc}
\hline \hline Workspace type & $S F W$ & $W F W$ & $D F W$ & $I D F W$ \\
\hline Covered Volume of the CDPR & $99.32 \%$ & $79.25 \%$ & $63.27 \%$ & $47.95 \%$ \\
\hline \hline
\end{tabular}

as shown in Fig. 4(b). Neglecting the effects of the external wrenches and the Coriolis forces, the volume of the DFW is $32 \%$ larger than the the volume of the improved DFW.

Similarly, by neglecting the inertia of the CDPR and taking into account only the external wrenches $\mathbf{w}_{e}$, the WFW occupies the $79.25 \%$ of the CDPR volume. By taking into account only the weight of the moving platform, the SFW covers $99.32 \%$ of the CDPR volume. These results are summarized in Tab. 1.

\section{Conclusion}

This paper introduced an improved dynamic feasible workspace for cable-driven parallel robots. This novel workspace takes into account: (i) The inertia of the moving platform; (ii) The external wrenches applied on the moving platform and (iii) The centrifugal and the Coriolis forces induced by a constant moving platform twist. As an illustrative example, the static, wrench-feasible, dynamic and improved dynamic workspaces of a spatial suspended cable-driven parallel robot, with the dimensions of a prototype developed in the framework of the IRT JV CAROCA project, are traced. It turns out that the IDFW of the CDPR under study is respectively 1.32 times, 1.65 times and 2.07 times smaller than its DFW, WFW and SFW. 


\section{Acknowledgements}

This research work is part of the CAROCA project managed by IRT Jules Verne (French Institute in Research and Technology in Advanced Manufacturing Technologies for Composite, Metallic and Hybrid Structures). The authors wish to associate the industrial and academic partners of this project, namely, STX, DCNS, AIRBUS and CNRS.

\section{References}

1. Barrette, G., Gosselin, C.: Determination of the dynamic workspace of cable-driven planar parallel mechanisms. ASME J. of Mech. Design 127(2), 242-248 (2005)

2. Bosscher, P., Riechel, A., Ebert-Uphoff, I.: Wrench-feasible workspace generation for cabledriven robots. IEEE Trans. on Robotics 22(5), 890-902 (2006)

3. Bouchard, S., Gosselin, C.M., Moore, B.: On the ability of a cable-driven robot to generate a prescribed set of wrenches. In: Proc. of the ASME Int. Design Eng. Tech. Conf. \& Comput. and Inform. in Eng. Conf. (IDETC/CIE 2008), pp. 47-58. Brooklyn, NY (2008)

4. Fattah, A., S.K., A.: Workspace and design analysis of cable-suspended planar parallel robots. In: Proc. of the ASME Int. Design Eng. Tech. Conf. \& Comput. and Inform. in Eng. Conf. (IDETC/CIE 2002), pp. 1095-1103. Montreal, QC (2002)

5. Gagliardini, L., Caro, S., Gouttefarde, M., Girin, A.: Discrete reconfiguration planning for cable-driven parallel robots. Mechanism and Machine Theory 100, 313 - 337 (2016). DOI http://dx.doi.org/10.1016/j.mechmachtheory.2016.02.014. URL http://www.sciencedirect.com/science/article/pii/S0094114X16000513

6. Gagliardini, L., Caro, S., Gouttefarde, M., Wenger, P., Girin, A.: A reconfigurable cable-driven parallel robot for sandblasting and painting of large structures. In: Cable-Driven Parallel Robots, Mechanisms and Machine Science, vol. 32, pp. 275-291. Springer (2015)

7. Gouttefarde, M., Collard, J.F., Riehl, N., Baradat, C.: Geometry selection of a redundantly actuated cable-suspended parallel robot. IEEE Transactions on Robotics 31(2), 501-510 (2015). DOI 10.1109/TRO.2015.2400253

8. Gouttefarde, M., Daney, D., Merlet, J.P.: Interval-analysis-based determination of the wrenchfeasible workspaceof parallel cable-driven robots. IEEE Trans. on Robotics 27(1), 1-13 (2011)

9. Gouttefarde, M., Gosselin, C.: Analysis of the wrench-closure workspace of planar parallel cable-driven mechanisms. IEEE Trans. on Robotics 22(3), 434-445 (2006)

10. Gouttefarde, M., Krut, S.: Advances in Robot Kinematics, chap. Characterization of parallel manipulator available wrench set facets, pp. 475-484. Springer (2010)

11. Guay, F., Cardou, P., Cruz, A., Caro, S.: Measuring how well a structure supports varying external wrenches. In: New Advances in Mechanisms, Transmissions and Applications, Mechanisms and Machine Science, vol. 17, pp. 385-392. Springer (2014)

12. Kozlov, V.: A graphical user interface for the design of cable-driven parallel robots. Master's thesis, Ecole Centrale de Nantes (2014)

13. Pusey, J., Fattah, A., Agrawal, S., Messina, E.: Design and workspace analysis of a 6-6 cablesuspended parallel robot. Mechanism and Machine Theory 39(7), 761-778 (2004)

14. Roberts, R., Graham, T., Lippitt, T.: On the inverse kinematics, statics, and fault tolerance of cable-suspended robots. J. of Robotic Syst. 15(10), 581-597 (1998)

15. Stump, E., Kumar, V.: Workspaces of cable-actuated parallel manipulators. ASME J. of Mech. Design 128(1), 159-167 (2006)

16. Verhoeven, R., Hiller: Advances in Robot Kinematics, chap. Estimating the controllable workspace of tendon-based Stewart platforms, p. 277284. Springer (2000) 